\title{
Selectively targeting proteins for degradation
}

\begin{abstract}
Ligand-induced target protein degradation has emerged as a promising therapeutic strategy, particularly in cancer, but efforts so far have often been limited by various factors, including poor target specificity and low potency. Now, writing in Science, Bradner and colleagues describe a novel chemical strategy to potently and specifically degrade target proteins, which they harness to delay leukaemia progression in mice.

Phthalimide-based drugs such as thalidomide, which have been successfully repurposed for the treatment of multiple myeloma, have recently been shown to bind to cereblon (CRBN; part of an ubiquitin E3 ligase complex). This promotes the recruitment of two transcription factors (IKZF1 and IKZF3) that
\end{abstract}

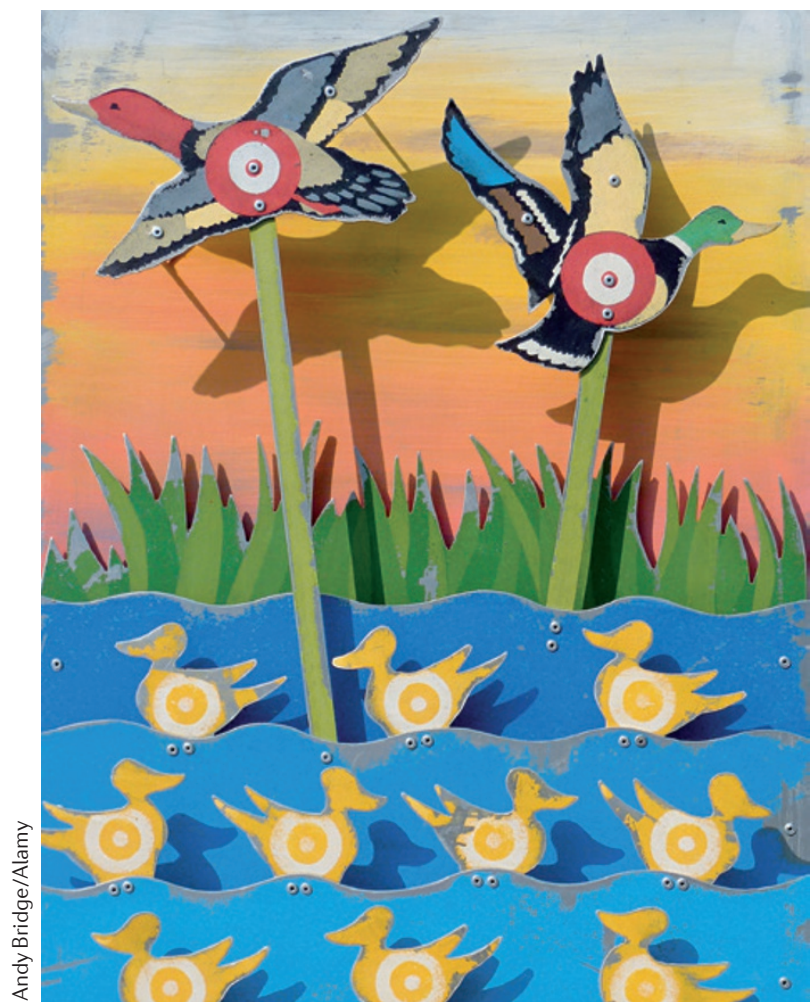

are essential to disease progression, resulting in their drug-induced ubiquitylation and degradation by the proteasome. Inspired by the finding that phthalimides bind to an E3 ligase, Bradner and colleagues hypothesized that rational design of phthalimide-conjugated ligands could promote CRBN-dependent target protein degradation. To test this, they focused on the BET bromodomain protein BRD4 - a transcriptional coactivator that regulates the expression of genes that promote cancer cell proliferation and survival. They conjugated their previously developed competitive BET bromodomain inhibitor, JQ1, with a novel thalidomide analogue, and the resulting molecule was named dBET1.

Selectivity profiling confirmed potent and BET-specific target engagement, and the crystal structure of ABET1 bound to BRD4 revealed that the mode of molecular recognition was comparable to JQ1. Using the dBET1-BRD4 crystal structure and the recently reported CRBNthalidomide structure, they modelled the feasibility of ternary complex formation, showing that $\mathrm{dBET} 1$ was capable of bridging BRD4 and CRBN without steric hindrance.

Next, the authors assessed the effect of dBET1 in cells. In a human acute myeloid leukaemia (AML) cell line (MV4;11), 2 hours of dBET1 treatment induced complete CRBN-dependent proteosomal degradation of BRD4. Compared with JQ1, dBET1 induced an enhanced apoptotic response in MV4;11 cells, DHL4 lymphoma cells and primary blasts from patients with leukaemia, demonstrating that such target degradation may exert a more potent effect than target inhibition.
Importantly, dBET1 was highly selective: out of 7,429 proteins, only the expression of the oncoproteins MYC and PIM1 (targets of JQ1), as well as BRD2, BRD3 and BRD4 was significantly downregulated.

In vivo, in a mouse hind limb xenograft model of human MV4;11 leukemia cells, 14 days of daily intraperitoneal dBET1 treatment substantially attenuated tumour progression and decreased tumor weight, in conjunction with BRD4 destabilization and MYC downregulation. Notably, dBET1 was well tolerated, exerting no changes in weight or complete blood counts. Moreover, in an aggressive disseminated leukemia mouse model (mCherry ${ }^{+}$MV4;11) with established disease, 19 days of dBET1 treatment resulted in a greater decrease in leukemic burden in bone marrow than did JQ1.

Finally, the authors assessed whether this strategy could be extended to other protein targets. Indeed, phthalimide-conjugated ligands designed to the cytosolic signaling protein FKBP12 (dFKBP12), potently decreased FKBP12 abundance in a human AML cell line.

In summary, given that CRBN is ubiquitously expressed in tissues, this strategy may have broad therapeutic utility, with the potential to overcome drug resistance and target previously intractable proteins. dBET1 and dFKBP12 are currently undergoing lead optimization for therapeutic development in both cancer and non-malignant diseases.

Sarah Crunkhorn

ORIGINAL RESEARCH PAPER Winter, G. et al. Phthalimide conjugation as a strategy for in vivo target protein degradation. Science http://dx.doi. org/10.1126/science.aab1433 